\section{THE AURORA OF MARCH 7}

To THE EDITOR OF ScIENCE: as a matter of record it may be worth while, even at this late date, to note that the aurora of March 7 was seen in Winter Park, Florida (latitude about $\left.28^{\circ} 37^{\prime}\right)$. It was visible for a short time only, between $9: 30$ and 10:00, Central Standard time. Those who saw it described the sky as brilliantly red for perhaps forty degrees along the northern horizon, with streamers extending half way to the zenith.

Winter Park, Fla.,

Frank P. Whitman

April 5, 1918

\section{SCIENTIFIC BOOKS}

Principes de Géométrie Analytique. Par Gaston Darboux. Gauthier-Villars et $\mathrm{C}^{\mathrm{ie}}$ 1917. Pp. vi +517 .

This important work has elements of interest extending beyond the circle of the professional mathematicians. It was the last mathematical contribution of one of the most noted French scientists and constituted the subject matter of his last course of lectures at the Sorbonne, closing a very successful teaching career which extended over a period of more than fifty years.

The principles of analytic geometry treated in this work relate mainly to the imaginary and the infinite in algebraic geometry, and hence they are also of great philosophic interest. In his Introduction the author states that these principles are too much neglected at the present time, being usually treated in the elementary courses where they can not be developed with the completeness which they merit and which he is free to give them here.

In our American text-books these principles are commonly omitted altogether. Comparatively few students become familiar with such interesting properties as those exhibited, for instance, by the two lines whose equation in rectangular coordinates is $x^{2}+y^{2}=0$. Each of these two lines is perpendicular to itse'f and has the property that the distance between any two of its points corresponding to finite coordinates is zero.
Our students of analytic geometry meet such equations as $x^{2}+y^{2}+1=0$, which are not satisfied by the coordinates of any real point. They are usually told that these equations represent imaginary curves, but if they consult some more advanced works; e. g., the Encyclopédie des Sciences Mathématiques tome III., volume 3 , page 260 , they find that what they commonly called imaginary circles and imaginary ellipses in their courses in analytic geometry are here called real curves. A real curve being one whose equation has real coefficients and hence does not need to contain any real point according to these authorities.

These remarks may serve to exhibit the facts that the imaginary in analytic geometry presents views which are quite different from those obtained by the student who confines himself to the consideration of real points, and that authorities do not agree as regards the definition of a real curve when the degree of the curve exceeds unity. Moreover, it is only necessary to recall the two circular points at infinity, which lie on all the circles of the plane, in order to remind ourselves of the fact that infinity also presents matters of interest which escape those who deal only with the finite region.

The volume under review is divided into five books with the following headings: anharmonic ratio, metric definitions, the theorems of Poncelet, Cayleyan geometry, and inversion. It has much in common with a work published by the same author under the title: "Sur une classe remarquable de courbes et de surfaces," 1872, but it contains many later developments. In particular, the part on Cayleyan geometry was developed by the author, according to the preface, during the years 1895 and 1896.

The book is not intended for the beginner in analytic geometry but presupposes some knowledge of this subject. Its chief aim seems to be to lay a solid foundation for the study of the imaginary and the infinite in geometry, and to present the subject in an attractive and simple manner with a view 